\title{
Short-Term Alcohol Abstinence Improves Antibacterial Defenses of Chronic Alcohol- Consuming Mice against Gut Bacteria-Associated Sepsis Caused by Enterococcus faecalis Oral Infection
}

\author{
Makiko Kobayashi, ${ }^{*}$ Akira Asai, ${ }^{\dagger}$ Ichiaki Ito, ${ }^{*}$ Sumihiro Suzuki, ${ }^{\ddagger}$ Kazuhide Higuchi, ${ }^{\dagger}$ and Fujio Suzuki ${ }^{\star}$
}

From the Department of Internal Medicine, * The University of Texas Medical Branch, Galveston, Texas; the Second Department of Internal Medicine, ${ }^{\dagger}$ Osaka Medical College, Takatsuki, Osaka, Japan; and the Department of Biostatistics and Epidemiology, ${ }^{\ddagger}$ University of North Texas Health Science Center, Fort Worth, Texas

Accepted for publication

May 9, 2017.

Address correspondence to Fujio Suzuki, Ph.D., Department of Internal Medicine, The University of Texas Medical Branch, 301 University Blvd, Galveston, TX 77555-0435. E-mail: fsuzuki@utmb.edu.

\begin{abstract}
The effects of short-term alcohol abstinence on host antibacterial resistance against Enterococcus faecalis oral infection was investigated in chronic alcohol-consuming mice [mice with $0.1 \mathrm{~g} /$ day of $20 \%$ ethanol consumption for 12 or 16 weeks (CAC-mice)]. These mice were highly susceptible to the infection; however, after 7 days of alcohol abstinence (aaCAC-mice), their antibacterial resistances were completely restored to the normal mouse level. Normal mice inoculated with CAC-mouse hepatic macrophages were shown to be susceptible to the infection, whereas the same macrophage preparation from aaCAC-mice did not impair the antibacterial resistance of normal mice. aaCAC-mouse liver macrophages protected nonobese diabetic-severe combined immunodeficiency IL-2R $\gamma^{\text {null }}$ mice exposed to E. faecalis, whereas those from CAC-mice did not. Monocyte-derived (MD) M2b macrophages were predominantly isolated from CAC-mouse livers, but these cells were not significantly isolated from aaCAC-mouse livers. Hepatic MD macrophages from aaCAC-mice switched to M1 macrophages in response to bacterial antigen, whereas the same macrophage preparation from CAC-mice did not. M1 Kupffer cells, M2a Kupffer cells, and MD M2b macrophages were shown to be not bactericidal, whereas $E$. faecalis was killed effectively by M1 macrophages derived from aaCAC-mouse hepatic MD macrophages. These results indicate that MD M2b macrophages predominantly distributed in the liver are responsible for the impaired resistance of CAC-mice to $E$. faecalis oral infection, and aaCAC-mice without MD M2b macrophages in the livers are resistant to the infection. (Am J Pathol 2017, 187: 1998-2007; http:// dx.doi.org/10.1016/j.ajpath.2017.05.013)
\end{abstract}

In the United States, 15.1 million adults (aged 18 years and older, $6.2 \%$ ) have an alcohol use disorder, including alcohol abuse and alcohol dependence. ${ }^{1}$ Nearly 88,000 individuals die from alcohol-related problems each year, making alcohol the fourth leading preventable cause of death in the United States. ${ }^{1}$ In addition, individuals with alcohol use disorders have an increased risk of serious infectious complications. ${ }^{2-6}$ Chronic alcohol consumption causes mucosal damage (increased intestinal permeability), intestinal dysbiosis (microbiota perturbations, including overgrowth of microbiota in the small intestine because of the alcohol-induced suppression of $\operatorname{Reg} 3 \mathrm{~b}$ and $\operatorname{Reg} 3 \mathrm{~g}$ antimicrobial protein production), and the dysfunctions of immune cells. These phenomena play a role on the bacterial translocation from intestinal tract to the liver, which frequently occurs in individuals with alcohol use disorders. ${ }^{6-10}$

For controlling sepsis caused by bacteria translocated from the intestine, macrophages located in the bacterial translocation sites (liver and mesenteric lymph nodes) have been characterized as a pivotal cell on host defense antibacterial

Supported by The University of Texas Medical Branch (Education, Research and Development).

Disclosures: None declared. 
effector cells. ${ }^{11-13}$ Because the liver is the primary site of alcohol metabolism, ${ }^{14}$ the function and properties of hepatic macrophages are greatly influenced by the degree of alcohol consumption. ${ }^{15-17}$ In the livers of individuals who consume small to moderate amounts of alcohol (up to one drink per day for women and up to two drinks per day for men), hepatic stellate cells and lymphocytes are activated, and resident tissue macrophages in the liver are polarized to the M1 phenotype (M1 Kupffer cells), stimulated by soluble factors involved in inflammatory responses. ${ }^{15-17}$ Although sustained inflammation is detrimental to the liver, inflammationassociated liver damage is generally resolved by M2a Kupffer cells. ${ }^{18,19}$ Thus, homeostasis between inflammatory and antiinflammatory responses is maintained in the livers of individuals who consume light to moderate amounts of alcohol through the balance of M1 Kupffer cells and M2a Kupffer cells. However, years of heavy alcohol consumption cause serious health problems, including severe chronic liver inflammation and serious infectious complications. ${ }^{20}$ Therefore, abstinence from alcohol has long been prescribed as the best way to avoid the risk of infectious complications in individuals who abuse alcohol. ${ }^{20}$ However, complete abstinence is difficult for these individuals without going to the hospital or after being discharged from the hospital. ${ }^{21-23} \mathrm{On}$ the basis of this background, a question arose whether the risk of infectious complications in individuals with alcohol abuse is removable without hospitalization by the cyclic regimen alternating between a brief time (less than a week) of alcohol abstinence and alcohol reconsumption. In this study, the effect of short-term abstinence on the host antibacterial resistance was investigated in chronic alcohol-consuming mice (CAC-mice; a model of individuals with alcohol use disorders). This approach leads to a more pragmatic regimen for removing the risk of infectious complications in individuals with alcohol abuse.

In our previous studies, CAC-mice were shown to be susceptible to Klebsiella pneumoniae intratracheal infection and Enterococcus faecalis oral infection. ${ }^{12}$ The major effector cells on the host antibacterial resistance against Klebsiella pneumonia and E. faecalis translocation were shown to be the M1 phenotype of macrophages (M1 macrophages) located at the infection sites (lung and mesenteric lymph nodes). However, the M2b phenotype of macrophages [M2b macrophages; $\mathrm{IL}-10^{+}$chemokine (C-C motif) ligand (CCL) $1^{+} \mathrm{CD} 163^{+}$], which suppress host antibacterial defenses against pneumonia and sepsis stemming from bacterial translocation, was predominated in these organs of CAC-mice ${ }^{12}$ and the peripheral blood of individuals with alcohol abuse. ${ }^{13}$ The switchability of these macrophages to the M1 phenotype is low, even though they are stimulated with antigens. ${ }^{11-13}$ The mortalities associated with pneumonia and gut bacteria-associated sepsis in CAC-mice were greatly mitigated through the modulation of M2b macrophages. ${ }^{12}$

In this study, the effect of short-term abstinence in the properties and function of hepatic macrophages in CAC-mice was studied, because the liver is not only an inflammation site organ influenced by chronic alcohol consumption but also a bacterial translocation site organ from the intestine. Two populations of macrophages have been described in the inflamed liver: Kupffer cells and macrophages derived from circulating monocytes (MD macrophages). ${ }^{24}$ Kupffer cells $\left(\mathrm{CD} 68^{+} \mathrm{F} 4 / 80^{+}\right.$cells $)$are fully differentiated resident macrophages in the liver, whereas MD macrophages $\left(\mathrm{CD} 11 \mathrm{~b}^{+} \mathrm{F} 4 /\right.$ $80^{+}$cells) are cells infiltrated from the circulation to the inflammation sites of the liver. ${ }^{24}$ In the results obtained, sepsis caused by E. faecalis oral infection was not developed in CAC-mice with short-term abstinence from alcohol (aaCACmice), although sepsis developed severely in CAC-mice exposed to the pathogen. The antibacterial responses of CAC-mice to E. faecalis oral infection were not directly influenced by M1 Kupffer cells and M2a Kupffer cells. MD macrophages were shown to be involved in the antibacterial properties of CAC-mice (MD-M2b macrophages) and aaCAC-mice (MD-M1 macrophages). The polarization of hepatic MD-M2b macrophages to interchangeable macrophages may cause the improved resistance of individuals who abuse alcohol against gut bacteria-associated sepsis. Shortterm abstinence is one of the ways to polarize MD-M2b macrophages to interswitchable macrophages.

\section{Materials and Methods}

Mice

The 9- to 12-week-old pathogen-free male and female BALB/ $\mathrm{c}$ mice and nonobese diabetic-severe combined immunodeficiency IL-2R $\gamma^{\text {null }}$ mice (NSG mice) were purchased from The Jackson Laboratory (Bar Harbor, ME). Mice with chronic ethanol consumption (CAC-mice) were prepared in $\mathrm{BALB} / \mathrm{c}$ mice by daily oral gavage of $0.5 \mathrm{~mL}$ of $20 \%$ ethanol $(0.1 \mathrm{~g} /$ day $)$ for 12 or 16 weeks. ${ }^{12}$ These mice were used in each experiment with (test groups) or without (control groups) short-term alcohol abstinence. Normal mice were given saline in the same manner and used as an additional control group. Also, both BALB/c mice and NSG mice were used as recipients in adoptive transfer experiments. The Institutional Animal Care and Use Committee of The University of Texas Medical Branch at Galveston approved all procedures using animals (approval number 1204024).

\section{Bacteria, Reagents, and Media}

Enterococcus faecalis (29212 strain), purchased from ATCC (Manassas, VA), was grown in trypticase soy broth for 18 hours at $37^{\circ} \mathrm{C}$ in aerobic conditions before being used in the infection experiments. ${ }^{11,12}$ Phosphatidylethanolamineconjugated anti-mouse F4/80 monoclonal antibody (mAb) was obtained from eBioscience (San Diego, CA). Phosphatidylethanolamine/Cy7-conjugated anti-mouse CD68, phosphatidylethanolamine/Cy5-conjugated anti-mouse CD11b, fluorescein isothiocyanate (FITC)-conjugated anti-mouse CCL1, FITC-conjugated anti-mouse CD206 
(mannose receptor), streptavidin particles plus-DM, cytofix/ cytopermsolution, isotype control antibodies, and IMag buffer were purchased from BD Biosciences (San Jose, CA).

\section{Preparation and Characterization of Hepatic} Macrophages (Kupffer Cells and MD Macrophages)

Mononuclear cells were isolated from the livers of normal mice, $12 \mathrm{~W}$ CAC-mice, and 12W CAC-mice 1 day after being subjected to 7 days of alcohol abstinence by collagenase digestion, followed by centrifugation on Percoll, as previously described. ${ }^{25}$ Obtained hepatic mononuclear cells were stained with biotinconjugated anti-mouse F4/80 mAb and allophycocyanin-conjugated anti-mouse CD68, phosphatidylethanolamine-conjugated anti-mouse $\mathrm{CD} 11 \mathrm{~b} \mathrm{mAb}$, FITC-conjugated anti-CD206 (mannose receptor) $\mathrm{mAb}$, or FITC-conjugated anti-CD38 mAb. Then, cell preparations were fixed in $2 \%$ paraformaldehyde. For the intracellular staining, $\mathrm{F} 4 / 80^{+}$cells were permeabilized with a Cytofix/Cytoperm solution at $4^{\circ} \mathrm{C}$ for 20 minutes, washed with a Perm/Wash solution, and then stained with FITC-conjugated anti-mouse CCL1 mAb or FITC-conjugated anti-mouse IL-12 mAb. CD206 ${ }^{+}$cells (M2a phenotype) and IL- $12^{+}$cells (M1 phenotype) in the $\mathrm{CD} 68^{+} \mathrm{F} 4 / 80^{+}$cell preparation (Kupffer cells) and $\mathrm{CCL}^{+}$cells (M2b phenotype) in the $\mathrm{CD} 11 \mathrm{~b}^{+} \mathrm{F} 4 / 80^{+}$cell preparation (MD macrophages) were analyzed by BD LSRFortessa (BD Biosciences) and FlowJo software version 10.3 (Tree Star, Inc., San Carlos, CA). Also, $\mathrm{CD}^{+} 8^{+} \mathrm{F} 4 / 80^{+}$cells, $\mathrm{CD} 68^{+} \mathrm{F} 4 / 80^{+} \mathrm{CD} 38^{+}$cells (M1 Kupffer cells), and CD11 b ${ }^{+} \mathrm{F} 4 /$ $80^{+}$cells were sorted from liver mononuclear cells from CACmice with or without abstinence by FACSAria IIU (BD Biosciences). In some experiments, total RNA containing miRNA was isolated from MD macrophages using the mirVana miRNA Isolation Kit (Ambion, Austin, TX), and cDNAs were prepared from miRNA using the TaqMan Advanced miRNA cDNA Synthesis Kit (Applied Biosystems, Foster City, CA). miR-27a expression levels were quantified by the ViiA 7 real-time PCR system (Applied Biosystems) using TaqMan Advanced miRNA Assays (Applied Biosystems). Data were normalized by miR361 expression levels and indicated mean fold change in $\Delta \Delta \mathrm{Ct}$ values as compared to control mice.

\section{Sepsis Stemming from E. faecalis Oral Infection}

Normal mice and 12W CAC-mice were used in these experiments throughout. Also, 16W CAC-mice were used in the first series of experiments. In addition, normal mice inoculated with hepatic macrophages from $12 \mathrm{~W}$ CAC-mice with or without short-term alcohol abstinence were used. Sepsis was developed in decontaminated and lansoprazole (a protonpump inhibitor)-treated mice after oral infection with $E$. faecalis, as previously described. ${ }^{11-13}$ In CAC-mice with decontamination and treatment with proton-pump inhibitor, E. faecalis-induced sepsis was reproducibly developed after oral infection with $10^{6}$ colony-forming units (CFUs)/mouse of $E$. faecalis. For decontamination, mice were given drinking water containing $4 \mathrm{mg} / \mathrm{mL}$ each of penicillin, streptomycin, and bacitracin for all day long $\times 4$ days. ${ }^{12}$ This procedure achieves transitory intestinal decontamination in mice for 7 to 10 days. On the day of the final antibiotic treatment, mice were treated orally with lansoprazole $(0.5 \mathrm{mg} / \mathrm{mL})$ to stabilize oral infection. ${ }^{11-13}$ Two days after lansoprazole treatment, these mice were infected orally with $10^{6} \mathrm{CFUs} /$ mouse of E. faecalis. This oral dose of E. faecalis corresponds to 0.01 $\mathrm{LD}_{50}$ in normal mice and $20 \mathrm{LD}_{50}$ in $12 \mathrm{~W}$ CAC-mice. The severity of infection was evaluated by the mortality rates (using $12 \mathrm{~W}$ and $16 \mathrm{~W}$ CAC-mice), and bacterial growth in mesenteric lymph nodes and liver (using 12W CAC-mice) after alcohol abstinence (test groups), in comparison with the corresponding CAC-mice (control groups) and normal mice (an additional control group). To determine the percentage of survival, mice were monitored twice a day for 2 weeks after infection. The numbers of bacteria in the organs of mice were determined by the colony counting method, as previously described. ${ }^{11-13}$ Sepsis stemming from E. faecalis translocation was not developed in normal mice, and all of the normal mice exposed to the pathogen in this manner survived for $>60$ days.

\section{Phagocytosis and Bactericidal Activities of Kupffer Cells and MD Macrophages}

To determine the percentage of phagocytic cells in Kupffer cells and MD macrophages from the livers of CAC-mice with or without abstinence, these cells were adjusted to $2 \times 10^{5}$ cells per well in an 8-well Lab-Tek II chamber slide (Nalgen Nunc International, Naperville, IL). Control M1 macrophages, M [lipopolysaccharide (LPS)/interferon (IFN)- $\gamma$ ], a reproducible experimental standard M1 macrophage, were bone marrow-derived macrophages that were previously cultured for 7 days with L929-conditioned medium and stimulated with 100 $\mathrm{ng} / \mathrm{mL}$ LPS and $20 \mathrm{ng} / \mathrm{mL} \mathrm{IFN-} \gamma$ for 48 hours, as previously described. ${ }^{26}$ These cells cultured in a Lab-Tek chamber slide were exposed to E. faecalis $\left(2 \times 10^{6} \mathrm{CFUs} / \mathrm{well}\right)$ for $30 \mathrm{mi}-$ nutes. Then, wells were washed twice with phosphate-buffered saline to remove non-phagocytosed bacteria, fixed, and stained with Wright-Giemsa stain. Macrophages that phagocytized five or more pathogens were microscopically counted in 200 adherent cells. The result was expressed as a percentage.

The bactericidal activities of Kupffer cells and MD macrophages were assayed by culturing test cells $\left(1 \times 10^{5}\right.$ cells/well) with $E$. faecalis $\left(3 \times 10^{5} \mathrm{CFUs} /\right.$ well $)$ at $37^{\circ} \mathrm{C}$ for 3 hours in antibiotic-free RPMI 1640 media supplemented with $10 \%$ fetal bovine serum. As a control, the pathogen was incubated alone. Three hours after incubation with the pathogen, cells were lysed in $0.1 \%$ Triton-X (SigmaAldrich, St. Louis, MO). Serial 10-fold dilutions of these fluids were plated on tryptic soy agar. The number of colonies was counted after being incubated for 24 hours at $37^{\circ} \mathrm{C}$. The following formula was applied to the results: ${ }^{12,13}$

$$
(1 \text { - test group CFUs/control group CFUs }) \times 100
$$

In some experiments, control M1 macrophages $\left(1 \times 10^{6}\right.$ cells $/ \mathrm{mL}$, lower chamber) obtained above were transwell 
cultured with MD macrophages $\left(5 \times 10^{5}\right.$ cells $/ \mathrm{mL}$, upper chamber) from CAC-mice with or without abstinence. Twenty-four hours after the cultivation, cells harvested from the lower chamber of the transwells were adjusted to $1 \times$ $10^{5}$ cells/well in antibiotic-free RPMI 1640 media supplemented with $10 \%$ fetal bovine serum, and exposed to $3 \times$ $10^{5} \mathrm{CFUs} /$ well of E. faecalis. Three hours after the cultivation, the bactericidal activities of M1 macrophages influenced by Kupffer cells or MD macrophages from CACmice with or without abstinence were determined, as described above.

\section{Adoptive Transfer of Macrophage Preparations}

In the adoptive transfer experiments, $\mathrm{F} 4 / 80^{+}$cells isolated from the livers of CAC-mice and aaCAC-mice were adjusted to $2 \times 10^{7}$ cells $/ \mathrm{mL}$ with phosphate-buffered saline, and 0.25 $\mathrm{mL}$ of the cell suspension was injected i.v. to normal mice or NSG mice that were previously decontaminated and treated with a proton-pump inhibitor, as described in Sepsis Stemming from E. faecalis Oral Infection. Macrophages adoptively transferred to NSG mice have been described to be spread throughout the whole body of recipient mice within 2 days of the inoculation. ${ }^{27}$ Two days after the cell inoculation, these mice were orally infected with $10^{6} \mathrm{CFUs} /$ mouse of $E$. faecalis. The effect of adoptively transferred cells on host antibacterial defenses in recipient mice to E. faecalis oral infection was evaluated by the mortality rates 2 weeks after infection.

\section{Statistical Analysis}

Data are presented as means \pm SEM. Results were statistically analyzed by a $t$-test. Survival rates were compared using Kaplan-Meier curves and the log-rank test. The result was considered significant if $P<0.05$.

\section{Results}

Short-Term Alcohol Abstinence Improves the Antibacterial Resistance of CAC-Mice to Sepsis Stemming from $E$. faecalis Oral Infection

To determine the effect of short-term abstinence on the antibacterial resistance of CAC-mice, $12 \mathrm{~W}$ and $16 \mathrm{~W}$ CACmice were subjected to 7-day alcohol abstinence and orally

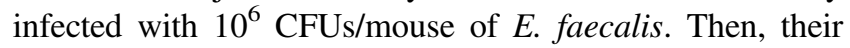
survival rates were compared to those of $12 \mathrm{~W}$ and $16 \mathrm{~W}$ CAC-mice without abstinence after exposure to the same infection. Normal mice that received saline were used as an additional control. Although $100 \%$ of normal mice and $12 \mathrm{~W}$ CAC-mice with alcohol abstinence survived 2 weeks or more after the infection, all 12W CAC-mice without abstinence died within 7 days of the infection (Figure 1A). Similar results were obtained when 16W CAC-mice were subjected to 7-day alcohol abstinence (Figure 1B). The effect of 7-day alcohol abstinence on the improved host antibacterial resistance was reproduced by the bacterial growth in the mesenteric lymph nodes, livers, and kidneys of 12W CAC-mice (Figure 1C). Thus, 2 days after the infection, the significant growth of the pathogen was not seen in these organs of normal mice and 12W CAC-mice with alcohol abstinence. However, $10^{5} \mathrm{CFUs/organ}$ or more of pathogen were detected in the livers of nonabstinent 12W CAC-mice 2 days after infection (Figure 1C). These results indicate that the antibacterial defense of $12 \mathrm{~W}$ and $16 \mathrm{~W}$ CAC-mice against sepsis caused by $E$. faecalis oral infection improves after short-term alcohol abstinence. Next, we examined the effect of various days of alcohol abstinence on the antibacterial resistance of $12 \mathrm{~W}$ CAC-mice against E. faecalis oral infection. Five days or more of alcohol abstinence were shown to be required for $12 \mathrm{~W}$ CAC-mice to improve host antibacterial resistance against sepsis stemming from E. faecalis translocation (Figure 1D). Therefore, all of the following experiments were performed in 12W CAC-mice with 7 days of alcohol abstinence (aaCAC-mice).

Hepatic Macrophages from CAC-Mice, but Not aaCACMice, Inhibit the Antibacterial Defenses of Recipient Mice to Sepsis Caused by E. faecalis Oral Infection

In our previous studies, M1 macrophages located in the bacterial translocation site tissues have been characterized as host defense effector cells against infectious complications caused by E. faecalis translocation. ${ }^{11,12}$ Also, M2b macrophages have been identified as suppressor cells for antibacterial effector cells. ${ }^{11-13}$ To determine the differences in the antibacterial functions of hepatic macrophages isolated from CAC-mice and aaCAC-mice, macrophages (F4/80 ${ }^{+}$ cells, a mixture of Kupffer cells and MD macrophages) were isolated from the livers of CAC-mice or aaCAC-mice, and adoptively transferred to normal mice orally infected with $10^{6} \mathrm{CFUs} / \mathrm{mouse}$ of E. faecalis. This amount of the pathogen corresponds to $0.01 \mathrm{LD}_{50}$ in normal mice and $20 \mathrm{LD}_{50}$ in CAC-mice. In the results, all of the normal mice inoculated with hepatic macrophages from CAC-mice died within 7 days of the infection. However, control normal mice and normal mice inoculated with hepatic macrophages from aaCAC-mice resisted the infection (Figure 2A). In addition, the bacterial growth greatly increased in the mesenteric lymph nodes of normal mice inoculated with hepatic macrophages from CAC-mice, whereas it did not in the same mice inoculated with hepatic macrophages from aaCACmice (Figure 2A). These results indicate that macrophages derived from CAC-mice are responsible for the increased susceptibility of normal mice to E. faecalis translocation, whereas macrophages derived from aaCAC-mice do not. Similar experiments were performed using NSG recipient mice infected with the pathogen. NSG mice lack functional lymphocytes, natural killer cells, and macrophages. All of the NSG mice inoculated with or without hepatic 
A

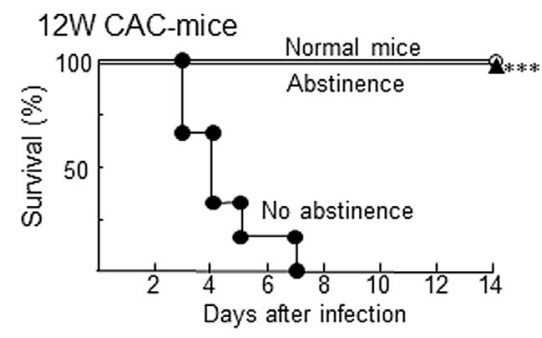

C

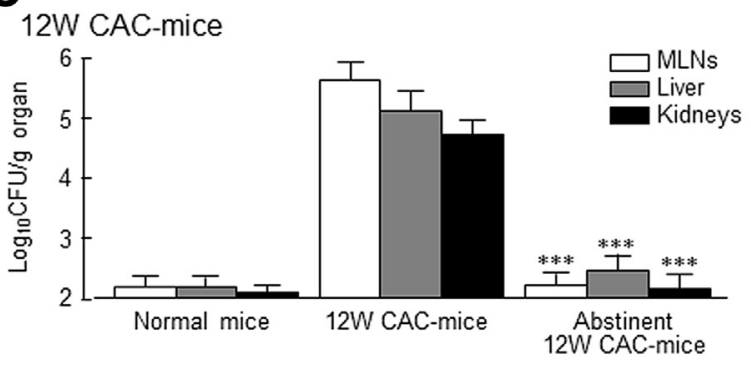

B

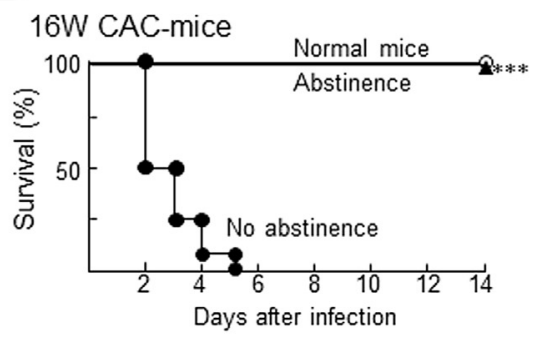

D 12W CAC-mice

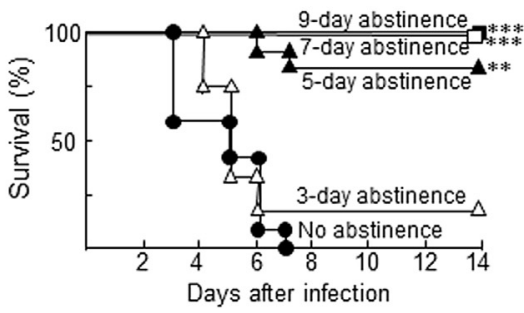

Figure 1 Short-term abstinence from alcohol improves the antibacterial resistance of $12 \mathrm{~W}$ and $16 \mathrm{~W}$ CAC-mice to sepsis stemming from $E$. faecalis oral infection. A and B: Sepsis was induced by E. faecalis oral infection $\left(10^{6}\right.$ colony-forming units/mouse) in 12 W CAC-mice $(\mathbf{A})$ and 16 W CAC-mice (B) with or without abstinence. As an additional control, normal mice given saline $(0.2 \mathrm{~mL} / \mathrm{mouse})$ in the same manner were infected with the pathogen. All mice were observed every 12 hours for 2 weeks to determine their mortalities. Data are representative of at least two independent experiments. Statistical significance was calculated from a log-rank test. C: The numbers of bacteria in organ homogenates of normal mice and 12W CAC-mice with or without abstinence were determined 2 days after $E$. faecalis oral infection. Data are representative of at least two independent experiments. D: Sepsis was induced by $E$. faecalis oral infection $\left(10^{6}\right.$ colony-forming units $/$ mouse) in 12W CAC-mice with abstinence for $0,3,5,7$, or 9 days. All mice were observed every 12 hours for 2 weeks to determine their mortalities. Data are representative of at least two independent experiments. Statistical significance was calculated from a log-rank test. Mice used in all experiments were decontaminated and treated with proton-pump inhibitor. Data are expressed as means \pm SEM (C). $n=12$ mice per group (A, B, and D); $n=5$ mice (C). ${ }^{* *} P<0.01,{ }^{* * *} P<0.001$ versus CAC-mice without abstinence. MLN, mesenteric lymph node; W, week.

macrophages from CAC-mice died within 5 days of the infection; however, $100 \%$ of the NSG mice inoculated with hepatic macrophages from aaCAC-mice survived after the same infection (Figure 2B). These results indicate that macrophages from the livers of CAC-mice and aaCAC-mice are functionally different.

Kupffer Cells Are Not Directly Involved in the Antibacterial Defenses of CAC-Mice with or without Short-Term Alcohol Abstinence

To determine the effect of short-term abstinence on the properties of resident macrophages in the livers of CACmice, $\mathrm{CD} 68^{+} \mathrm{F} 4 / 80^{+}$cells (Kupffer cells) were sorted from hepatic mononuclear cells of normal mice, CAC-mice, or aaCAC-mice, and analyzed for the expression of IL-12 and CD206 by flow cytometry. IL-12 is a biomarker of M1 macrophages, and CD206 is a biomarker of M2a macrophages. In the results, the numbers of $\mathrm{CD} 206^{+}$cells (M2a Kupffer cells) did not significantly change in the livers of normal mice (resistant mice to sepsis stemming from $E$. faecalis translocation), CAC-mice (susceptible mice to the infection), and aaCAC-mice (resistant mice to the infection) (Figure 3A). This indicates that the development of sepsis stemming from $E$. faecalis translocation in CAC-mice is not directly influenced by M2a Kupffer cells. On the other hand, the numbers of $\mathrm{IL}-12^{+}$cells (M1 Kupffer cells) increased greatly in the livers of CAC-mice, and these cells decreased to the level shown by normal mice in the livers of aaCACmice (Figure 3B). Next, we examined the phagocytic functions and bactericidal activities of M1 Kupffer cells $\left(\mathrm{CD} 68^{+} \mathrm{F} 4 / 80^{+} \mathrm{CD} 38^{+}\right.$cells) sorted from liver mononuclear cells. In the results, $56 \%$ of M1 Kupffer cells were shown to be phagocytized cells. However, these cells were not able to kill the pathogen effectively (Figure 3C). Similarly, M1 Kupffer cells from aaCAC-mice were shown to be cells with phagocytic functions and without bactericidal activities. This series of experiments indicates that the antibacterial defenses of aaCAC-mice against E. faecalis oral infection are not directly influenced by M2a and M2 Kupffer cells.

\section{Short-Term Alcohol Abstinence Decreases the Numbers of MD-M2b Macrophages in the Livers of CAC-Mice}

To determine a role of short-term abstinence on the properties of MD macrophages in the livers of CAC-mice, $\mathrm{CD} 11 \mathrm{~b}^{+} \mathrm{F} 4 / 80^{+}$cells (MD macrophages) were sorted from hepatic mononuclear cells of normal mice and CAC-mice with or without abstinence, and analyzed for the expression of CCL1 by flow cytometry. CCL1 is a biomarker of M2b macrophages. In the results, the numbers of $\mathrm{CD} 11 \mathrm{~b}^{+} \mathrm{F} 4 / 80^{+} \mathrm{CCL}^{+}{ }^{+}$cells (MD-M2b macrophages) were 

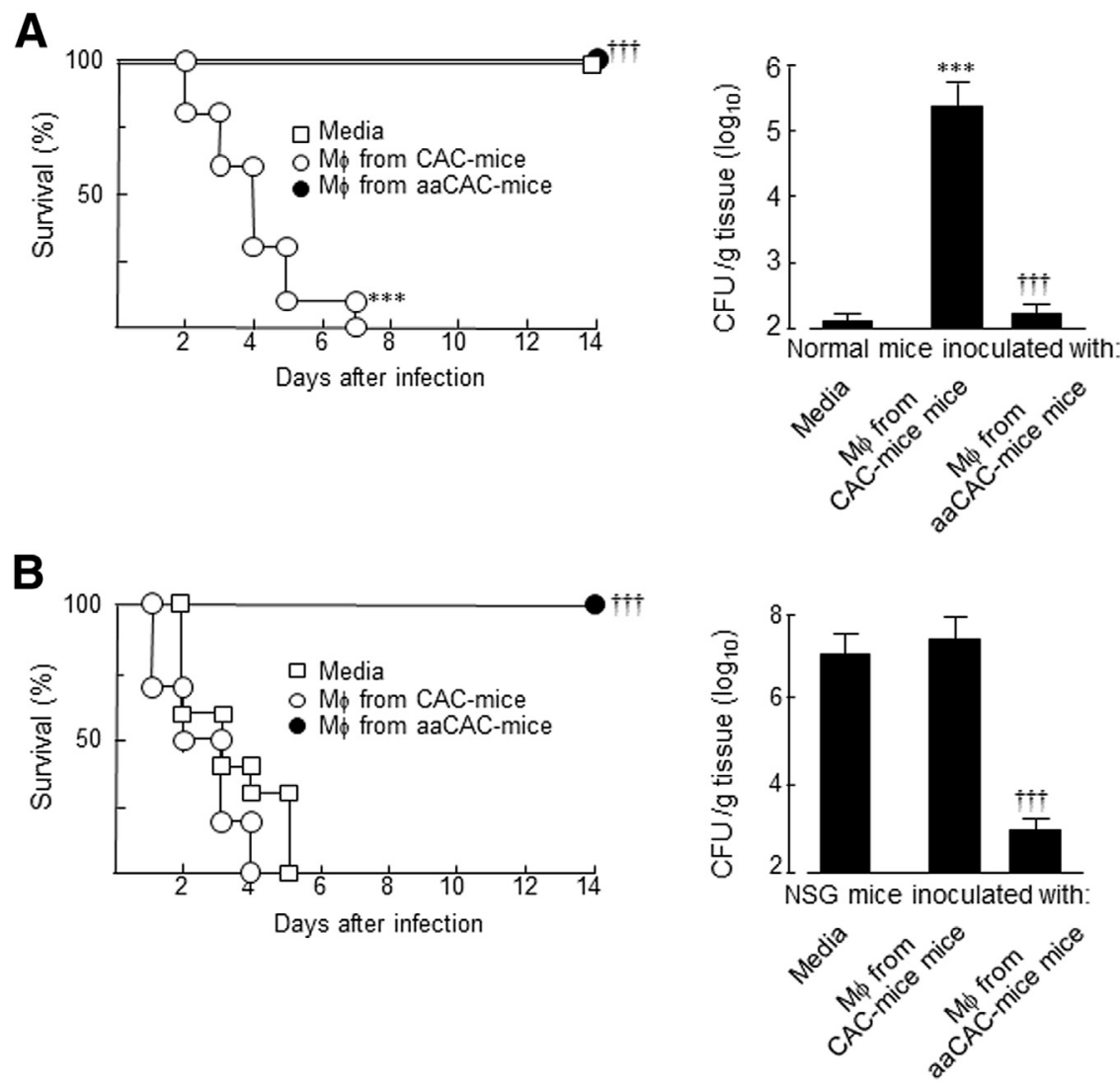

Figure 2 Host antibacterial resistance influenced by hepatic macrophages $(M \varphi)$ from CACmice and aaCAC-mice. Normal mice (A) and NSG mice (B) were i.v. inoculated with $\mathrm{F} 4 / 80^{+}$cells $\left(5 \times 10^{6}\right.$ cells/mouse) from the liver of CAC-mice or aaCAC-mice. Then, they were orally infected with $E$. faecalis $\left[10^{6}\right.$ colony-forming units (CFUs)/ mouse]. The same recipients treated with media served as a control. Survival: All mice were observed every 12 hours for 2 weeks to determine their mortalities. Data are displayed by percentage survival. Data are representative of at least two independent experiments. Statistical significance was calculated from a log-rank test. Bacterial growth: Mesenteric lymph nodes obtained from recipient mice 2 days after $E$. faecalis oral infection were homogenized. The number of bacteria in each organ homogenate was determined by colony counting. Data are representative of at least two independent experiments. All experiments were performed in mice after decontamination and treatment with proton-pump inhibitor. Data are expressed by the means \pm SEM. $n=3$ to 5 mice (A and B, bacterial growth); $n=10$ mice (A and B, survival). ${ }^{* *} P<0.001$ versus recipients treated with media; ${ }^{\dagger \dagger} P<0.001$ versus recipient mice inoculated with $\mathrm{F} 4 / 80^{+}$cells from CAC-mice. greatly increased in the livers of CAC-mice, but not in aaCAC-mice (Figure 4A). This was confirmed by the decreased expression of miR-27a, a specific biomarker of M2b macrophages (Figure 4B). In the next experiments, the function of MD-M2b macrophages from the livers of CACmice with or without abstinence was examined based on the inhibitory activity against M1 macrophage-associated bactericidal activities. As a control, M1 macrophages, M(LPS/IFN- $\gamma$ ), a standard M1 macrophage, were generated from bone marrow-derived macrophages after stimulation with $100 \mathrm{ng} / \mathrm{mL}$ LPS and $20 \mathrm{ng} / \mathrm{mL} \mathrm{IFN-} \gamma$ for 48 hours, as previously described. ${ }^{26} \mathrm{M}(\mathrm{LPS} / \mathrm{IFN}-\gamma)$ were cultured in the lower chamber of the transwells. In the results, M(LPS/IFN- $\gamma$ ) transwell cultured with CAC-mouse hepatic MD macrophages did not display any bactericidal activities. However, the bactericidal activity of M(LPS/IFN- $\gamma$ ) was not influenced in transwell cultures performed with MD macrophages from the livers of aaCAC-mice (Figure 4C). These results indicate that short-term abstinence is effective in decreasing the numbers and suppressor cell functions of MD-M2b macrophages in the livers of CAC-mice. In addition, M1 macrophages were induced by the bacterial antigen in cultures of MD macrophages isolated from the livers of aaCAC-mice. MD macrophages from aaCAC-mice displayed strong bactericidal activities and produced IL-12 in cultures supplemented with heat-killed E. faecalis (Figure 4D). However, MD macrophages from CAC-mice were not able to kill the pathogen and did not produce IL-
12 in their cultures added with the antigen. These results indicate that MD-M1 macrophages readily appear in the livers of aaCAC-mice with bacterial translocation.

\section{Discussion}

Individuals with alcohol use disorder have an increased risk of infectious complications stemming from gut microbiota. ${ }^{6-10}$ Abstinence from alcohol has long been described as the best way to avoid the risk of serious infectious complications and many other health problems associated with alcohol abuse. ${ }^{20}$ However, alcohol abstinence is difficult for individuals with alcohol use disorder to sustain..$^{21,22}$ In an 8-year perspective study of 1162 individuals entering various alcohol treatment programs with a $94 \%$ follow-up rate, only $36 \%$ of the individuals who were abstinent for less than a year remained abstinent. ${ }^{21}$

In the present study, we investigated the effect of shortterm alcohol abstinence on the antibacterial resistance of CAC-mice against sepsis stemming from E. faecalis oral infection. In the results, we found that sepsis did not develop in CAC-mice subjected to 7 days of alcohol abstinence (aaCAC-mice) after oral infection with E. faecalis, whereas sepsis developed easily in CAC-mice exposed to the same infection. Although normal mice are resistant to this infection, infectious complications were developed by E. faecalis oral infection in normal mice adoptively transferred with 
A
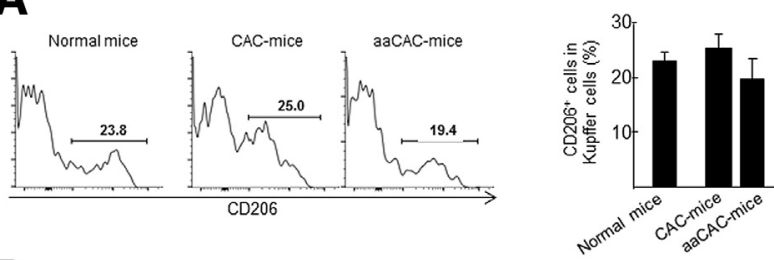

B
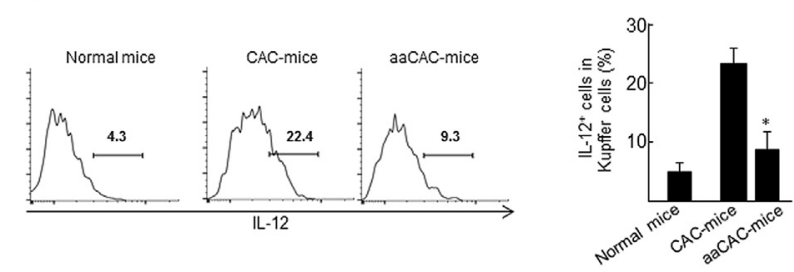

C

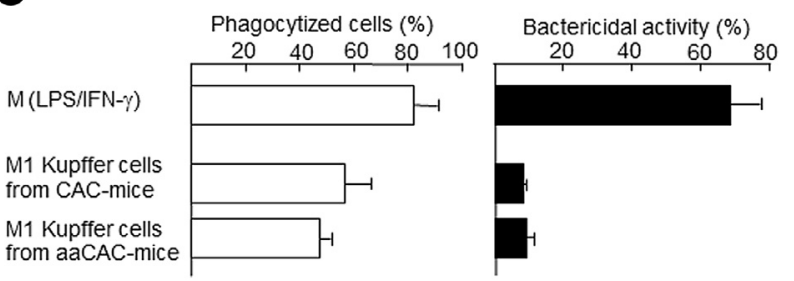

Figure 3 Properties of Kupffer cells from the livers of CAC-mice and aaCAC-mice. $\mathbf{A}$ and $\mathbf{B}$ : Liver mononuclear cells from CAC-mice and aaCACmice were analyzed for $\mathrm{CD} 8^{+} \mathrm{F} 4 / 80^{+} \mathrm{CD}^{206^{+}}$(M2a Kupffer cells; $\mathrm{A}$ ) or $\mathrm{CD} 68^{+} \mathrm{F} 4 / 80^{+} \mathrm{IL}-12^{+}$(M1 Kupffer cells; B) by flow cytometry. The same cells derived from normal mice served as an additional control. Data shown in left panels are representative of three independent experiments. The obtained results were combined and displayed in right panels as the percentages of $\mathrm{CD}_{206}{ }^{+}$cells (A) or IL- $12^{+}$cells (B) in the liver $\mathrm{CD} 68^{+} \mathrm{F} 4 / 80^{+}$ cells of these mice. C: $\mathrm{CD} 68^{+} \mathrm{F} 4 / 80^{+} \mathrm{CD} 38^{+}$cells (M1 Kupffer cells) were sorted from the hepatic mononuclear cells of CAC-mice and aaCAC-mice, and tested for their phagocytosis and bactericidal activities. Control M1 macrophages were standard M1 macrophages generated from bone marrow-derived macrophages after stimulation with $100 \mathrm{ng} / \mathrm{mL}$ lipopolysaccharide (LPS) and $20 \mathrm{ng} / \mathrm{mL}$ interferon (IFN)- $\gamma$ for 48 hours, as previously described $[M(L P S / I F N)] .^{23}$ Data are representative of at least two independent experiments. Data are expressed as means \pm SEM (A and $\mathbf{B}) . n=4$ to 6 mice $(\mathbf{A}$ and $\mathbf{B})$. ${ }^{*} P<0.05$ versus CAC-mice.

$\mathrm{F} 4 / 80^{+}$cells (macrophages including both Kupffer cells and MD macrophages) from CAC-mice. Normal mice adoptively transferred with the same macrophage preparation from aaCAC-mice were shown to be resistant against the infection. In our studies, MD macrophages isolated from the livers of aaCAC-mice were determined to be changeable macrophages to M1 macrophages. Therefore, macrophages adoptively transferred and distributed to bacterial translocation site tissues may play a role on the host antibacterial defenses against sepsis caused by E. faecalis oral infection. The numbers of M1 Kupffer cells increased in the livers of CAC-mice, but these cells were not shown to be bactericidal. The numbers of M2a Kupffer cells did not change in the liver of CAC-mice before (susceptible to sepsis) and after (resistant against sepsis) the alcohol abstinence. These results indicate that host antibacterial defenses of CAC-mice to E. faecalis oral infection are not directly influenced by
M1 Kupffer cells and M2a Kupffer cells. In contrast, a large number of MD-M2b macrophages were demonstrated in the livers of CAC-mice. The numbers of MD-M2b macrophages were reduced in the livers of aaCAC-mice to the level shown in those of normal mice. In response to the antigen stimulation, MD macrophages in the livers of aaCAC-mice readily switched to MD-M1 macrophages, whereas MD macrophages in the livers of CAC-mice did not switch to other phenotypes, even after being stimulated with the antigen. These results indicate that a brief period of alcohol abstinence causes the decreased distribution of MDM2b macrophages to the livers of CAC-mice.

Increased bacterial translocation in individuals with alcohol use disorders has been considered to be associated with alcohol-induced alternations in the intestine. ${ }^{28,29}$ During alcohol consumption, intestinal permeability is known to be increased, and it is resolved after abstinence from alcohol. ${ }^{29}$ In addition to alcohol consumption, increased intestinal permeability has been demonstrated in mice exposed to $5 \mathrm{~Gy}$ of whole body $\gamma$-irradiation (irradiated mice) because of radiation-induced intestinal damages. ${ }^{30,31}$ Our studies showed that irradiated mice were extremely susceptible to sepsis caused by $E$. faecalis oral infection. ${ }^{11}$ Similar to $12 \mathrm{~W}$ CAC-mice, M2b macrophages predominated in the bacterial translocation site tissues were shown to be responsible for the increased susceptibility of irradiated mice to infectious complications. ${ }^{11}$ After the modulation of bacterial translocation site $\mathrm{M} 2 \mathrm{~b}$ macrophages by treatment with a CCL1 inhibitor (CCL1 antisense oligodeoxynucleotide), irradiated mice became resistant against sepsis stemming from E. faecalis oral infection. ${ }^{11}$ An inhibitor of CCL1 does not function to improve intestinal permeability. These results indicate that the host antibacterial resistance in aaCAC-mice to E. faecalis oral infection is not directly influenced by the intestinal permeability.

In addition, it is well known that alcohol induces intestinal disbiosis. ${ }^{10,28}$ In our infection experiments, decontaminated mice were orally exposed to a constant amount of E. faecalis. The numbers of bacteria in the guts were shown to be $<10^{3}$ CFUs/g in mice 2 days after decontamination. This indicates that disbiosis influenced by alcohol consumption is not directly reflected in the results of our experiments. Additional analytical studies using naturally occurring bacterial translocation will be required to determine the contribution of disbiosis on the antibacterial resistance of CAC-mice subjected to a short-term abstinence.

Recently, miRNA directly induced by alcohol was shown to be a polarizer of quiescent monocytes to $\mathrm{M} 2 \mathrm{~b}$ monocytes. ${ }^{32}$ In our preliminary studies, M2b monocytes were detected in cultures of healthy donor peripheral blood monocytes after long-term exposure to $10 \mathrm{mmol} / \mathrm{L}$ of ethanol. Recently, the expression profile of miRNAs in M1 and M2a macrophages has been analyzed using microarray and quantitative RT-PCR array techniques. More specifically, miR-9, miR-127, miR-155, and miR-125b have been shown to promote M1 
A

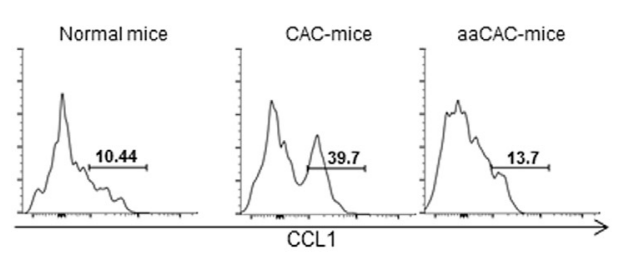

B
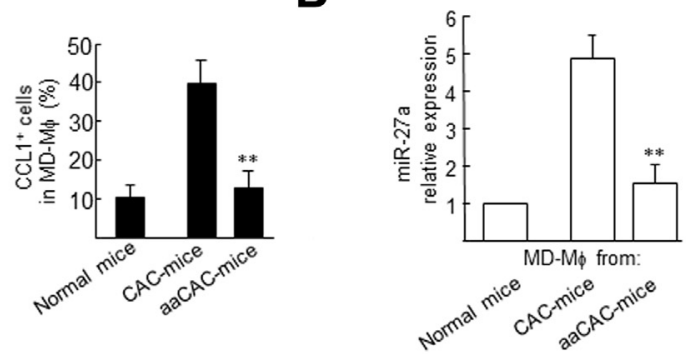

C

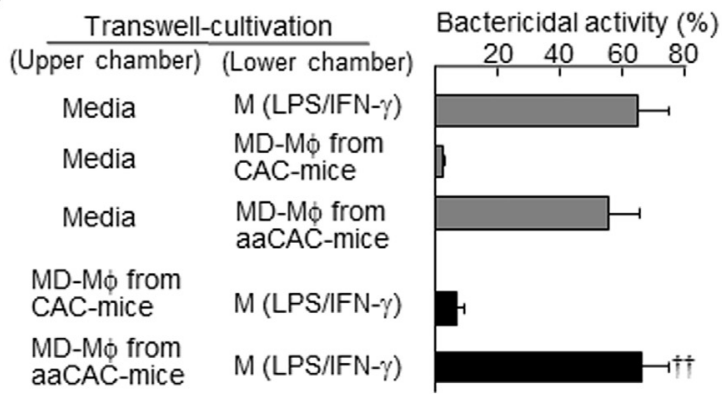

D

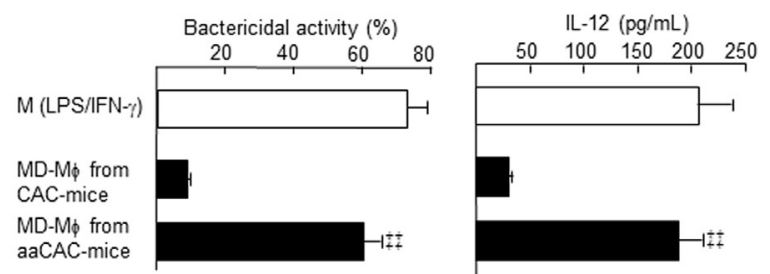

Figure 4 Properties of monocyte-derived macrophages (MD-M $\varphi$ ) from CAC-mice and aaCAC-mice. A: Liver mononuclear cells from CAC-mice and aaCAC-mice were analyzed for $\mathrm{CD} 11 \mathrm{~b}^{+} \mathrm{F} 4 / 80^{+} \mathrm{CCL} 1^{+}$(MD-M2b macrophages) by flow cytometry. The same cells derived from normal mice served as an additional control. Data shown in the left panels are representative of three independent experiments. The obtained results were combined in the right panel as the percentages of $\mathrm{CCL}^{+}$cells in the hepatic MD macrophages from these mice. B: $\mathrm{CD} 11 \mathrm{~b}^{+} \mathrm{F} 4 / 80^{+}$cells (MD macrophages), sorted from the liver mononuclear cells prepared from three groups of mice, were tested for miR-27a expression by real-time PCR. miR-27a is a typical biomarker for M2b macrophages. Data are displayed as relative expression. Data are representative of at least two independent experiments. C: The suppressor cell activity of MD macrophages, sorted from the livers of CAC-mice and aaCAC-mice, against control M1 macrophages was tested in transwell cultures. Control M1 macrophages were generated from bone marrow-derived macrophages after stimulation with $100 \mathrm{ng} / \mathrm{mL}$ lipopolysaccharide (LPS) and $20 \mathrm{ng} / \mathrm{mL}$ interferon (IFN)- $\gamma$ for 48 hours, as previously described $[M(L P S / I F N)] .{ }^{23}$ Control M1 macrophages (lower chambers) were transwell cultured with hepatic MD macrophages from CAC-mice or aaCAC-mice. Control M1 macrophages and hepatic MD macrophages from CAC-mice and aaCAC-mice were cultured with media, and served as controls. Twenty-four hours after the cultivation, cells in the lower chamber were tested for their bactericidal activities. Data are representative of at least two independent experiments. D: MD macrophages $\left(\mathrm{CD} 11 \mathrm{~b}^{+} \mathrm{F} 4 / 80^{+}\right.$cells, $1 \times 10^{6}$ cells $\left./ \mathrm{mL}\right)$, sorted from the livers of CAC-mice or aaCAC-mice, were tested for their bactericidal activities (left panel). Also, these cells stimulated with the antigen were cultured for 24 hours, and culture fluid harvested was assayed for IL-12 by enzyme-linked immunosorbent assay (right panel). LPS/IFN- $\gamma$-induced M1 macrophages from bone marrow-derived macrophages served as a positive control. Data are representative of at least two independent experiments. Data are expressed as means \pm SEM (A, right panel, and $\mathbf{B})$. $n=4$ to 6 mice $(\mathbf{A}$, right panel); $n=5$ mice (B). ${ }^{* *} P<0.01$ versus CAC-mice; ${ }^{\dagger \dagger} P<0.01$ versus M1 macrophages transwell cultured with MD macrophages from the livers of CAC-mice; ${ }^{\ddagger \ddagger} P<0.01$ versus MD macrophages from the livers of CAC-mice.

polarization, whereas miR-124, miR-223, miR-34a, let-7c, miR-132, miR-146a, and miR-125a-5p induce M2a polarization. ${ }^{33}$ miR-27a has been described as a specific biomarker of M2b macrophages, ${ }^{33}$ and CD38 (M1) and Egr2 (M2) have been identified as new biomarkers for M1 and M2 macrophages. ${ }^{34}$ In our studies, miR-27a was detected in MD-M2b macrophages isolated from the livers of CAC-mice.

Liver diseases frequently develop in individuals with alcohol use disorders. It has been reported that M2 macrophages play an important role in controlling hepatocellular injuries (excessive or prolonged inflammation in liver) in ethanol-fed mice. ${ }^{35}$ In the damaged livers, Kupffer cells (fully differentiated resident macrophages) and MD macrophages (macrophages derived from circulating monocytes) are known to be coexistent. ${ }^{24,35}$ Recent fate-mapping experiments showed that adult Kupffer cells develop almost exclusively from yolk sac-derived macrophages that are distinct from hematopoietic stem cells. ${ }^{36-39}$ The development of yolk sac-derived macrophages is dependent on the transcription factor PU.1, whereas hematopoietic stem cell-derived macrophages are required for the transcription factor MYB. ${ }^{36-39}$ In this study, the numbers of MD-M2b macrophages (but not M2a Kupffer cells) decreased dramatically in the livers of aaCAC-mice. From these results, the question arose why the function of MD-M2b macrophages was affected by a short-term alcohol abstinence. Generally, macrophages sequentially change their functional phenotype in response to microenvironmental signals. ${ }^{40}$ However, M2b macrophages do not easily switch to another phenotype because of endogenous production of CCL1, an essential chemokine for their prolongation. ${ }^{41}$ In our previous studies, ${ }^{12,13} \mathrm{M} 2 \mathrm{~b}$ macrophages appearing in chronic alcohol-consuming hosts switched to the non-M2b phenotype when endogenous CCL1 production was modified by treatment with CCL1 antisense oligodeoxynucleotide. In our current studies, therefore, we are investigating whether CCL1 gene silencing occurs in M2b macrophages in CAC-mice after short-term alcohol abstinence. In the 
results, growth arrest-specific 5, a long noncoding RNA involved in $C C L 1$ gene silencing, ${ }^{42}$ was expressed by MD macrophages from the livers of normal mice and aaCACmice. However, the significant level of GAS5 was not demonstrated in the same macrophage preparation from CAC-mice. Furthermore, we examined what mechanisms are involved in the reduced GAS5 RNA levels in MD macrophages from CAC-mice, focusing on the nonsensemediated RNA decay (NMD) pathway. NMD is a key RNA metabolizing mechanism responsible for the rapid degradation of RNAs. ${ }^{43}$ UPF1 (a key molecular component of the NMD pathway) and other RNA-binding proteins interact with transcribed RNA. UPF1 is phosphorylated after bingeing to RNA to recruit RNase; this leads to the degradation of the GAS5 transcripts. ${ }^{44,45}$ Therefore, we examined UPF1 phosphorylation levels in MD macrophages from the livers of CAC-mice and aaCAC-mice by Western blotting. UPF1 phosphorylation was detected in MD macrophages from CAC-mice, but not in those from aaCAC-mice. From these results, the dephosphorylation or inhibiting phosphorylation of UPF1 may occur in MD macrophages from CAC-mice subjected to a brief period of abstinence. Also, high mobility group box 1 may be involved in NMD activation in CAC-mice, because high mobility group box 1 is released from necrotic cells in the inflamed tissues (such as livers) during chronic alcohol consumption and induces miR-222, which is involved in NMD activation. ${ }^{46} \mathrm{~A}$ brief time of abstinence may improve the abnormal high mobility group box $1 /$ miR-222 levels in CAC-mice. Further studies will be required.

\section{References}

1. National Institute on Alcohol Abuse and Alcoholism. Alcohol facts and statistics. Rockville, MD, NIAAA, 2016. Available at https://pubs. niaaa.nih.gov/publications/AlcoholFacts\&Stats/AlcoholFacts\&Stats. htm (accessed February 2017)

2. von Dossow V, Schilling C, Beller S, Hein OV, von Heymann C, Kox WJ, Spies CD: Altered immune parameters in chronic alcoholic patients at the onset of infection and of septic shock. Crit Care 2004, 8: R312-R321

3. Szabo G, Mandrekar P: A recent perspective on alcohol, immunity, and host defense. Alcohol Clin Exp Res 2009, 33:220-232

4. Boé DM, Vandivier RW, Burnham EL, Moss M: Alcohol abuse and pulmonary disease. J Leukoc Biol 2009, 86:1097-1104

5. Bhatty M, Pruett SB, Swiatlo E, Nanduri B: Alcohol abuse and Streptococcus pneumoniae infections: consideration of virulence factors and impaired immune responses. Alcohol 2011, 45:523-539

6. Tuomisto S, Pessi T, Collin P, Vuento R, Aittoniemi J, Karhunen PJ: Changes in gut bacterial populations and their translocation into liver and ascites in alcoholic liver cirrhotics. BMC Gastroenterol 2014, 14:40

7. Tabata T, Tani T, Endo Y, Hanasawa K: Bacterial translocation and peptidoglycan translocation by acute ethanol administration. J Gastroenterol 2002, 37:726-731

8. Wang L, Fouts DE, Stärkel P, Hartmann P, Chen P, Llorente C, DePew J, Moncera K, Ho SB, Brenner DA, Hooper LV, Schnabl B: Intestinal REG3 lectins protect against alcoholic steatohepatitis by reducing mucosa-associated microbiota and preventing bacterial translocation. Cell Host Microbe 2016, 19:227-239
9. Yan AW, Schnabl B: Bacterial translocation and changes in the intestinal microbiome associated with alcoholic liver disease. World J Hepatol 2012, 4:110-118

10. Vassallo G, Mirijello A, Ferrulli A, Antonelli M, Landolfi R, Gasbarrini A, Addolorato G: Alcohol and gut microbiota: the possible role of gut microbiota modulation in the treatment of alcoholic liver disease. Aliment Pharmacol Ther 2015, 41:917-927

11. Kobayashi M, Nakamura K, Cornforth M, Suzuki F: Role of M2b macrophages in the acceleration of bacterial translocation and subsequent sepsis in mice exposed to whole body ${ }^{137} \mathrm{Cs} \gamma$-irradiation. J Immunol 2012, 189:296-303

12. Ohama H, Asai A, Ito I, Suzuki S, Kobayashi M, Higuchi K, Suzuki F: M2b macrophage elimination and improved resistance of mice with chronic alcohol consumption to opportunistic infections. Am J Pathol 2015, 185:420-431

13. Tsuchimoto Y, Asai A, Tsuda Y, Ito I, Nishiguchi T, Garcia MC, Suzuki S, Kobayashi M, Higuchi K, Suzuki F: M2b monocytes provoke bacterial pneumonia and gut bacteria-associated sepsis in alcoholics. J Immunol 2015, 195:5169-5177

14. Zakhari S: Overview: how is alcohol metabolized by the body? Alcohol Res Health 2006, 29:245-254

15. Nelson S, Kolls JK: Alcohol, host defence and society. Nat Rev Immunol 2002, 2:205-209

16. Heymann F, Tacke F: Immunology in the liver: from homeostasis to disease. Nat Rev Gastroenterol Hepatol 2016, 13:88-110

17. Zeng T, Zhang CL, Xiao M, Yang R, Xie KQ: Critical roles of Kupffer cells in the pathogenesis of alcoholic liver disease: from basic science to clinical trials. Front Immunol 2016, 29:538

18. Laskin DL, Sunil VR, Gardner CR, Laskin JD: Macrophages and tissue injury: agents of defense or destruction? Annu Rev Pharmacol Toxicol 2011, 51:267-288

19. Wan J, Benkdane M, Teixeira-Clerc F, Bonnafous S, Louvet A, Lafdil F, Pecker F, Tran A, Gual P, Mallat A, Lotersztajn S, Pavoine C: M2 Kupffer cells promote M1 Kupffer cell apoptosis: a protective mechanism against alcoholic and nonalcoholic fatty liver disease. Hepatology 2014, 59:130-142

20. Addolorato G, Mirijello A, Barrio P, Gual A: Treatment of alcohol use disorders in patients with alcoholic liver disease. J Hepatol 2016, 65: 618-630

21. Dennis ML, Foss MA, Scott CK: An eight-year perspective on the relationship between the duration of abstinence and other aspects of recovery. Eval Rev 2007, 31:585-612

22. Jesse S, Bråthen G, Ferrara M, Keindl M, Ben-Menachem E, Tanasescu R, Brodtkorb E, Hillbom M, Leone MA, Ludolph AC: Alcohol withdrawal syndrome: mechanisms, manifestations, and management. Acta Neurol Scand 2017, 135:4-16

23. Schmidt KJ, Doshi MR, Holzhausen JM, Natavio A, Cadiz M, Winegardner JE: Treatment of severe alcohol withdrawal. Ann Pharmacother 2016, 50:389-401

24. Zigmond E, Samia-Grinberg S, Pasmanik-Chor M, Brazowski E, Shibolet O, Halpern Z, Varol C: Infiltrating monocyte-derived macrophages and resident Kupffer cells display different ontogeny and functions in acute liver injury. J Immunol 2014, 193:344-353

25. Ikarashi M, Nakashima H, Kinoshita M, Sato A, Nakashima M, Miyazaki H, Nishiyama K, Yamamoto J, Seki S: Distinct development and functions of resident and recruited liver Kupffer cells/macrophages. J Leukoc Biol 2013, 94:1325-1336

26. Zajac E, Schweighofer B, Kupriyanova TA, Juncker-Jensen A, Minder P, Quigley JP, Deryugina EI: Angiogenic capacity of M1- and M2-polarized macrophages is determined by the levels of TIMP-1 complexed with their secreted proMMP-9. Blood 2013, 122: 4054-4067

27. Shultz LD, Brehm MA, Garcia-Martinez JV, Greiner DL: Humanized mice for immune system investigation: progress, promise and challenges. Nat Rev Immunol 2012, 12:786-798

28. Leclercq S, Matamoros S, Cani PD, Neyrinck AM, Jamar F, Stärkel P, Windey $\mathrm{K}$, Tremaroli V, Bäckhed F, Verbeke K, de Timary $\mathrm{P}$, 
Delzenne NM: Intestinal permeability, gut-bacterial dysbiosis, and behavioral markers of alcohol-dependence severity. Proc Natl Acad Sci U S A 2014, 111:E4485-E4493

29. Leclercq S, Cani PD, Neyrinck AM, Stärkel P, Jamar F, Mikolajczak M, Delzenne NM, de Timary P: Role of intestinal permeability and inflammation in the biological and behavioral control of alcohol-dependent subjects. Brain Behav Immun 2012, 26:911-918

30. Yin L, Vijaygopal P, Menon R, Vaught LA, Zhang M, Zhang L, Okunieff P, Vidyasagar S: An amino acid mixture mitigates radiationinduced gastrointestinal toxicity. Health Phys 2014, 106:734-744

31. Shukla PK, Gangwar R, Manda B, Meena AS, Yadav N, Szabo E, Balogh A, Lee SC, Tigyi G, Rao R: Rapid disruption of intestinal epithelial tight junction and barrier dysfunction by ionizing radiation in mouse colon in vivo: protection by N-acetyl-1-cysteine. Am J Physiol Gastrointest Liver Physiol 2016, 310:G705-G715

32. Saha B, Bruneau JC, Kodys K, Szabo G: Alcohol-induced miR-27a regulates differentiation and M2 macrophage polarization of normal human monocytes. J Immunol 2015, 194:3079-3087

33. Graff JW, Dickson AM, Clay G, McCaffrey AP, Wilson ME: Identifying functional microRNAs in macrophages with polarized phenotypes. J Biol Chem 2012, 287:21816-21825

34. Jablonski KA, Amici SA, Webb LM, Ruiz-Rosado Jde D, Popovich PG, Partida-Sanchez S, Guerau-de-Arellano M: Novel markers to delineate murine M1 and M2 macrophages. PLoS One 2015, 10:e0145342

35. Wang M, You Q, Lor K, Chen F, Gao B, Ju C: Chronic alcohol ingestion modulates hepatic macrophage populations and functions in mice. J Leukoc Biol 2014, 94:657-665

36. Gomez Perdiguero E, Klapproth E, Schulz C, Busch K, Azzoni E, Crozet L, Garner H, Trouillet C, de Bruijn MF, Geissmann F,
Rodewald HR: Tissue-resident macrophages originate from yolk-sacderived erythro-myeloid progenitors. Nature 2015, 518:547-551

37. Italiani P, Boraschi D: New insights into tissue macrophages: from their origin to the development of memory. Immune Netw 2015, 15 : 167-176

38. Wang J, Kubes P: A reservoir of mature cavity macrophages that can rapidly invade visceral organs to affect tissue repair. Cell 2016, 165: 668-678

39. Ley K, Pramod AB, Croft M, Ravichandran KS, Ting JP: How mouse macrophages sense what is going on. Front Immunol 2016, 7:204

40. Sica A, Mantovani A: Macrophage plasticity and polarization: in vivo veritas. J Clin Invest 2012, 122:787-795

41. Asai A, Nakamura K, Kobayashi M, Herndon DN, Suzuki F: CCL1 released from $\mathrm{M} 2 \mathrm{~b}$ macrophages is essentially required for the maintenance of their properties. J Leukoc Biol 2012, 92:859-867

42. Cao Q, Wang N, Qi J, Gu Z, Shen H: Long non-coding RNA-GAS5 acts as a tumor suppressor in bladder transitional cell carcinoma via regulation of chemokine (C-C motif) ligand 1 expression. Mol Med Rep 2016, 13:27-34

43. Hug N, Longman D, Cáceres JF: Mechanism and regulation of the nonsense-mediated decay pathway. Nucleic Acids Res 2016, 44: $1483-1495$

44. Mourtada-Maarabouni M, Williams GT: Growth arrest on inhibition of nonsense-mediated decay is mediated by noncoding RNA GAS5. Biomed Res Int 2013, 2013:358015

45. Tani H, Torimura M, Akimitsu N: The RNA degradation pathway regulates the function of GAS5 a non-coding RNA in mammalian cells. PLoS One 2013, 8:e55684

46. Mari E, Zicari A, Fico F, Massimi I, Martina L, Mardente S: Action of HMGB1 on miR-221/222 cluster in neuroblastoma cell lines. Oncol Lett 2016, 12:2133-2138 